\title{
FORUM
}

\section{The Running Fix on an Ellipsoid}

\author{
Robin G. Stuart \\ (Valhalla, New York, USA) \\ (E-mail: robinstuart@earthlink.net)
}

\begin{abstract}
The running fix or sight-run-sight fix is a classic problem in celestial navigation. Methods employed to obtain the fix traditionally involve advancing a Line of Position (LoP) taken at an earlier time and crossing it with one obtained later. Attempts to generalise the operation of advancing an LoP when the Earth's surface is represented by a plane to the case of the sphere have resulted in proposals that contain poorly constrained approximations or are otherwise fundamentally flawed. A simple rapidly-convergent iterative procedure to obtain a running fix is described that avoids the notion of advanced LoPs and is readily applicable to both the sphere and ellipsoid.
\end{abstract}

\section{KE Y W ORD S}
1. Celestial Navigation.
2. Astronomical Running Fix.
3. Rhumb line.

1. INTRODUCTION. In classical celestial navigation a running fix is obtained by combining sights taken at two different times and positions. The relative distance and bearing of the two positions are assumed to be known from the ship's course and speed.

In practice such a fix is often obtained graphically by plotting Lines of Position (LoP) $\mathrm{LoP}_{1}$ and $\mathrm{LoP}_{2}$ associated with the first and second sights respectively on a nautical chart or plotting sheet. $\mathrm{LoP}_{1}$ is then translated or advanced by a distance and direction that reflects the rhumb line track of the vessel between the two observations. The point where the advanced $\mathrm{LoP}_{1}$ crosses $\mathrm{LoP}_{2}$ provides the running fix. This procedure generally works satisfactorily on small scales over which the Earth's surface can be represented by a plane and LoPs are well approximated by straight lines.

On the surface of a sphere an LoP obtained from a celestial sight becomes a Circle of Position (CoP) centred on the point on the Earth's surface where the observed celestial body is directly overhead. This is referred to as the body's Geographic Position (GP).

Attempts have been made to extend the notion of advancing an LoP to the case of the sphere with the idea that the running fix could then be found as the intersection point of two circles using double altitude or similar methods (Metcalf, 1991; Zevering, 2006). The problem, as has been pointed out previously (Williams, 1998; Huxtable, 2006), is that if each point on a $\mathrm{CoP}$ is individually advanced on a rhumb line course of specified distance and bearing, the resultant locus of points is no longer a circle. This point is illustrated in 


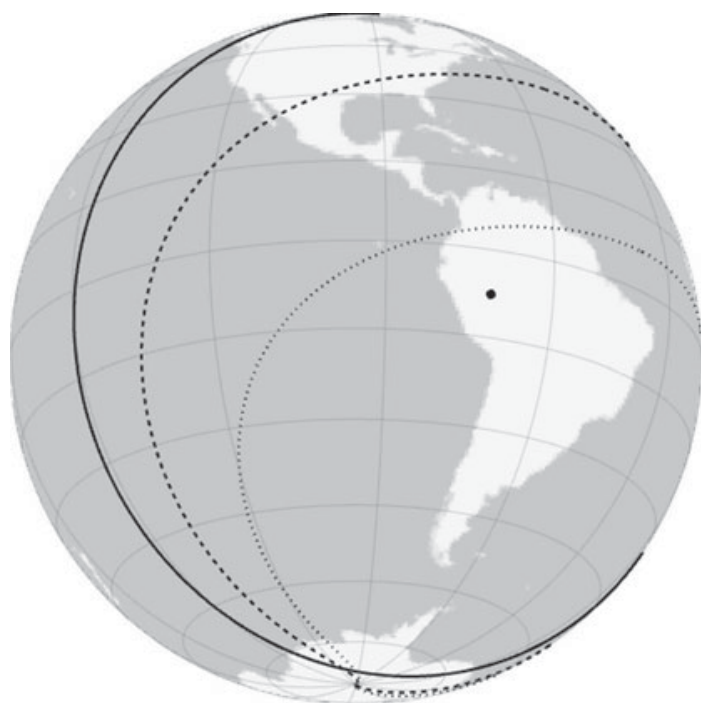

Figure 1. Effect of advancing each point on an initial Circle of Position indicated by the heavy curve by $2000 \mathrm{~nm}$ (dashed curve) and $4000 \mathrm{~nm}$ (dotted curve) on a course of $160^{\circ}$ True. The centre of the original $\mathrm{CoP}$ is shown. Large displacements have been chosen to make the resulting distortions clearly visible.

Figure 1. It follows that any approach that purports to obtain a running fix on the surface of a sphere as the intersection of two circles is fundamentally flawed.

An equation satisfied by points on the advanced $\mathrm{CoP}$ and its generalization to the ellipsoid has been given by Williams (1998) who suggests solving a pair of non-linear simultaneous equations to obtain the fix.

On reflection it is perhaps unfortunate that the graphical technique used on a plane for obtaining a running fix has so strongly coloured the thinking and influenced the approaches when it comes to a running fix on curved surfaces where advancing or transferring an LoP offers no obvious advantages.

In this note the problem of the running fix on the surface of the Earth is approached in a manner that avoids the need for advancing an LoP as a whole. Indeed the method will be described for the case of an ellipsoidal Earth as it is only marginally more complicated than the spherical case.

It is to be expected that, because of the complex mixture of logarithms and trigonometric functions that appear in Mercator sailing formulae, solutions will necessarily be numerical in nature even in the case of a sphere. It will be shown that the numerical problem can be reduced to one of finding the root of a function of just one variable and does not require solving simultaneous non-linear equations.

On an ellipsoid a celestial sight yields a set of possible positions that lie neither on a circle nor a line. In what follows LoP should considered to stand for Locus of Position.

2. RUNNING FIX ON THE SURFACE OF AN ELLIPSOID. Assume a sight of a celestial body is made when the ship is initially at a position denoted $P_{1}$. The sight yields the zenithal distance, $\mathrm{ZD}_{1}$, for the observed body. At the instant of observation the GP of the body is specified by its declination, $\delta_{1}$, and Greenwich Hour Angle, GHA 1 . From this 
information it can be determined that $P_{1}$ lies somewhere on a locus of position $\mathrm{LoP}_{1}$. The vessel then travels a distance, $D$, on a rhumb line course of bearing, $C$, measured east from north to a position denoted $P_{2}$ where a second sight is made. This sight produces a corresponding set of parameters $\mathrm{ZD}_{2}, \delta_{2}$ and $\mathrm{GHA}_{2}$ which determines that the vessel's position, denoted $P_{2}$, lies somewhere on $\mathrm{LoP}_{2}$.

The general running fix requires finding positions $P_{1}$ and $P_{2}$ that satisfy the following conditions

1) $P_{1}$ lies on $\mathrm{LoP}_{1}$

2) $P_{2}$ lies a distance $D$ from $P_{1}$ along a rhumb line course with bearing $C$.

3) $P_{2}$ lies on $\mathrm{LoP}_{2}$

The required fix is given by $P_{2}$. In general it is to be expected that two geographically widely separated sets of points can be found to satisfy these conditions. A similar situation arises in double altitude sights. A reasonable initial estimate of position ensures that the correct solution is selected.

Assume a value $L_{1}$ for the latitude of position $P_{1}$ and find the corresponding longitude, $\lambda_{1}$, such that $P_{1}$ lies on $\mathrm{LoP}_{1}$ using the equation

$$
\lambda_{1}=-\mathrm{GHA}_{1} \pm \cos ^{-1}\left(\frac{\cos Z D_{1}-\sin \delta_{1} \sin L_{1}}{\cos \delta_{1} \cos L_{1}}\right)
$$

The upper (lower) sign applies when the object lies to the observer's west (east). This formula follows from the familiar cosine rule of spherical trigonometry but because geodetic or astronomical latitude and longitude on the surface of an ellipsoid are defined from the direction of the normal to the surface it applies to both the sphere and ellipsoid. An explicit proof is given by Williams (1998, Section 9.3). On the sphere, points on the LoP all lie the same geodesic distance from the GP; however this is not true for an ellipsoid.

Compute the position $P_{2}$, with latitude $L_{2}$ and longitude, $\lambda_{2}$, located a distance $D$ along a rhumb line course of bearing $C$ from $P_{1}$. This is the direct Mercator sailing problem which can be solved, in principle, using the equations

$$
\begin{aligned}
& \mathrm{LP}\left(L_{2}\right)=\mathrm{LP}\left(L_{1}\right)+\left(\frac{D}{a}\right) \cos C \\
& \lambda_{2}=\lambda_{1}+\left(\mathrm{MP}\left(L_{2}\right)-\mathrm{MP}\left(L_{1}\right)\right) \tan C
\end{aligned}
$$

Here $a$ is the Earth's equatorial radius and $\operatorname{MP}(\phi)$ is the meridional part:

$$
\mathrm{MP}(\phi)=\ln \left(\left[\frac{1-e \sin \phi}{1+e \sin \phi}\right]^{\frac{e}{2}} \tan \left(\frac{\pi}{4}+\frac{\phi}{2}\right)\right)
$$

When $L_{2}=L_{1}$ Equation (2) becomes

$$
\lambda_{2}=\lambda_{1}+\left(\frac{D}{a}\right) \frac{\sqrt{1-e^{2} \sin ^{2} L_{1}}}{\cos L_{1}}
$$


The function $\operatorname{LP}(\phi)$ is the meridional arc length in units of $a$ from the equator to the latitude $\phi$ :

$$
L P(\phi)=\left(1-e^{2}\right) \int_{0}^{\phi}\left(1-e^{2} \sin ^{2} \theta\right)^{-\frac{3}{2}} d \theta
$$

This can be written in terms of Legendre elliptic integrals of the second or third kind:

$$
\operatorname{LP}(\phi)=E(\phi, e)-\frac{\frac{1}{2} e^{2} \sin 2 \phi}{\sqrt{1-e^{2} \sin ^{2} \phi}}=\left(1-e^{2}\right) \Pi\left(\phi, e^{2}, e\right)
$$

where conventions defined by Olver et al. (2010) have been used.

These functions are available in some standard software packages. Solving Equation (1) for $L_{2}$ requires evaluating both the function $\operatorname{LP}(\phi)$ and its inverse. Series expansions in the Earth's eccentricity, $e$, have been given by Tseng et al. (2012). To $O\left(e^{4}\right)$ their results can be written

$$
\begin{aligned}
& \mathrm{LP}(\phi)=\left(1-e^{2}\right)\left[M_{0} \phi-\left(\frac{3}{8} e^{2}+\frac{15}{32} e^{4}+\ldots\right) \sin 2 \phi+\left(\frac{15}{256} e^{4}+\ldots\right) \sin 4 \phi+\ldots\right] \\
& \operatorname{LP}^{-1}(\omega)=\mu+\left(\frac{3}{8} e^{2}+\frac{3}{16} e^{4}+\ldots\right) \sin 2 \mu+\left(\frac{21}{256} e^{4}+\ldots\right) \sin 4 \mu+\ldots
\end{aligned}
$$

where $M_{0}=\frac{2}{\pi} \frac{E(e)}{\left(1-e^{2}\right)}=1+\frac{3}{4} e^{2}+\frac{45}{64} e^{4}+\ldots$ and the rectifying latitude $\mu=\frac{\omega}{\left(1-e^{2}\right) M_{0}}$.

In the case of the sphere $\operatorname{LP}(\phi)=\phi$.

Having obtained $L_{2}$ and $\lambda_{2}$ for position $P_{2}$ from Equations (1) and (2) compute the quantity:

$$
\mathrm{f}=\sin \delta_{2} \sin L_{2}+\cos \delta_{2} \cos L_{2} \cos \left(\lambda_{2}+\mathrm{GHA}_{2}\right)-\cos Z D_{2}
$$

$P_{2}$ lies on $\operatorname{LoP}_{2}$ when $\mathrm{f}\left(L_{1}\right)=0$ and the three conditions listed above will all be satisfied. The starting value $L_{1}$ can be adjusted iteratively using standard methods for finding the root of a function of one variable. Convergence is expected to be rapid provided the intersection angle between the LoPs is not too small which is a standard requirement for reliable fixes.

In this procedure the path between $P_{1}$ and $P_{2}$ is a single rhumb line but it could equally be constructed from multiple rhumb line legs by the repeated application of Equations (1) and (2).

Practical limitations involved in following a precise rhumb line track mean that treating the running fix on the ellipsoid is likely to meet or exceed all real world requirements for accuracy. If it were necessary to go a step further and consider the geoid it would be most natural and efficient ${ }^{1}$ to compute a set of corrections to $P_{1}$ and/or $P_{2}$ once they have been determined by the method described above. Such additional corrections will not be considered here.

\footnotetext{
${ }^{1}$ Natural because the geoid is itself specified in terms of deviations from the reference ellipsoid and efficient because it is only necessary to consider the geoid in the regions near $P_{1}$ and $P_{2}$.
} 
3. AN EXAMPLE. In what follows WGS84 geodetic datum is assumed.

On 29 February 2016 a Sun sight taken at 17:00:00 UT from a ship near latitude $48^{\circ} \mathrm{N}$ yields $\mathrm{ZD}_{1}=77^{\circ} 36 \cdot 8^{\prime}$ and for which the Nautical Almanac gives $\mathrm{GHA}_{1}=71^{\circ} 54 \cdot 3^{\prime} ; \delta_{1}=$ $-7^{\circ} 36 \cdot 8^{\prime}$. The Sun's azimuth is $Z_{n}=117^{\circ}$.

Over the next five hours the vessel travels 50 nautical miles on a course of $160^{\circ}$ True at which point a second Sun sight gives $\mathrm{ZD}_{2}=56^{\circ} 13 \cdot 6^{\prime}$ with $\mathrm{GHA}_{2}=146^{\circ} 54 \cdot 9^{\prime}$; $\delta_{2}=-7^{\circ} 32 \cdot 1^{\prime}$.

For the purposes of illustration it will be assumed that these values are exact.

With these parameters $\mathrm{f}\left(47^{\circ} 30^{\prime}\right)=0.0103083$ and $\mathrm{f}\left(48^{\circ}\right)=0.0023569$. With these initial values the solution $\mathrm{f}\left(L_{1}\right)=0$ can be found by standard iterative methods such as the Secant Method (Press et al., 2007).

The table below gives the result of iterations performed until successive estimates for $P_{2}$ differ by less than 1 metre.

Table 1. Successive estimates of latitude. $L$, and longitude, $\lambda$, of positions $P_{1}$ and $P_{2}$ in solving the equation $\mathrm{f}\left(L_{1}\right)=0$. The column labelled $\Delta P_{2}$ gives the change in the location of $P_{2}$ compared to the previous estimate in metres.

\begin{tabular}{lcccccc}
\hline Iteration & $\mathrm{L}_{1}\left({ }^{\circ}\right)$ & $\lambda_{1}\left({ }^{\circ}\right)$ & $\mathrm{L}_{2}\left({ }^{\circ}\right)$ & $\lambda_{2}\left({ }^{\circ}\right)$ & $\mathrm{f}\left(L_{1}\right)$ & $\boldsymbol{\Delta} P_{2}(\mathrm{~m})$ \\
\hline 0 & 47.500000 & $-134 \cdot 116697$ & 46.717296 & -133.699422 & 0.0103083 & - \\
& 48.000000 & -133.748681 & 47.217364 & -133.327444 & 0.0023569 & - \\
\hline 1 & $48 \cdot 148207$ & -133.637668 & 47.365592 & -133.215237 & $-1.53 \times 10^{-4}$ & 18,537 \\
2 & $48 \cdot 147255$ & -133.638384 & 47.364640 & -133.215960 & $2.23 \times 10^{-8}$ & 119 \\
3 & $48 \cdot 147257$ & -133.638382 & 47.364642 & -133.215959 & $2.11 \times 10^{-13}$ & $0 \cdot 2$ \\
\hline
\end{tabular}

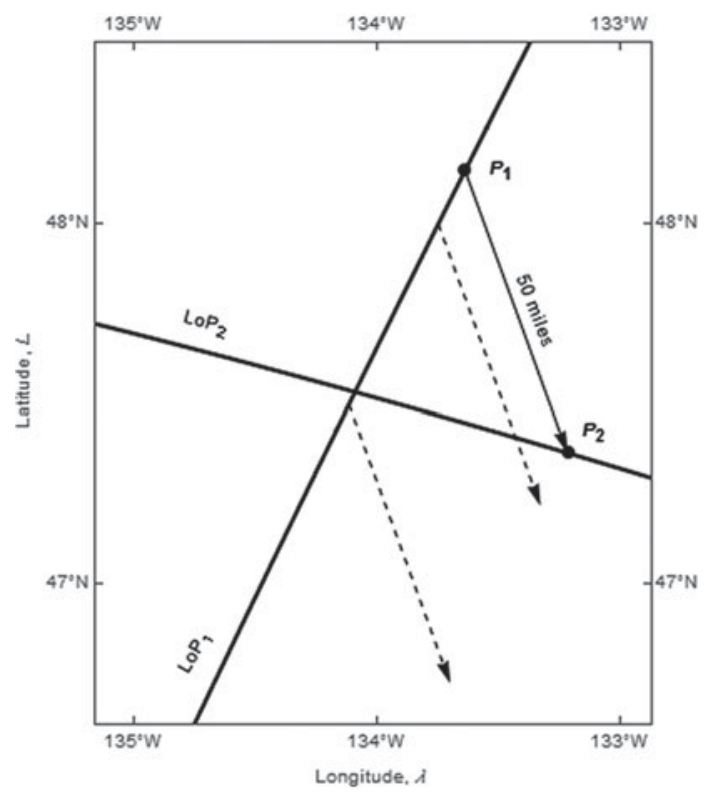

Figure 2. The iterative procedure described in the text finds points $P_{1}$ and $P_{2}$ lying on $\mathrm{LoP}_{1}$ and $\mathrm{LoP}_{2}$ respectively and separated by a specified rhumb line distance of (50 nautical miles) and bearing $\left(160^{\circ}\right.$ True). The dotted arrows are the initial trials used in Iteration 0 of Table 1 . These satisfy 1) and 2) of the conditions listed in the text but not 3 ). Since $\mathrm{LoP}_{1}$ is not advanced it does not undergo the distortion illustrated in Figure 1 and therefore retains its relatively simple algebraic form. 
An additional iteration changes the position of $P_{2}$ by $2 \mu \mathrm{m}$. The locations of $P_{2}$ obtained by assuming a sphere $(e=0)$ and WGS84 ellipsoid $(e=0.08181919 \ldots)$ differ by $4.4 \mathrm{~m}$. Convergence is rapid as the LoPs are fairly straight over the scale of this problem.

The result $P_{2}=47^{\circ} 21.878^{\prime} \mathrm{N}, 133^{\circ} 12.958^{\prime} \mathrm{W}$ is the required fix at the time of the second celestial sight. The vessel's position at the time of the first sight $P_{1}=48^{\circ} 08 \cdot 835^{\prime} \mathrm{N}$, $133^{\circ} 38.303^{\prime} \mathrm{W}$ is also obtained as a by-product without additional computational effort. The positions $P_{1}$ and $P_{2}$ satisfy the three conditions listed in the text are therefore consistent with all available information including the vessel's distance and direction of travel. A graphical representation of the results of the procedure is shown in Figure 2.

4. CONCLUSIONS. In a running fix two celestial sights and the known track of the vessel provide three key pieces of information or conditions that must be satisfied for the fix to be valid. It has been shown that the solution to this problem can be reduced to one of finding the root of a well-behaved function in one variable and that standard iterative methods can be applied to determine the root to arbitrary accuracy. The procedure yields the unique position that is consistent with all the available information and is applicable to both the sphere and ellipsoid. The need to advance the initial LoP with its associated complexities and ambiguities is avoided.

\section{REFERENCES}

Huxtable, G. (2006). An Erroneous Proposal To Allow For Travel Of An Observer Between Two Celestial Altitude Observations. The Journal of Navigation, 59, 521-529.

Metcalf, T.R. (1991). Advancing Celestial Circles of Position, NAVIGATION: Journal of the Institute of Navigation. 38, 285-288.

Olver, F.W.J., Lozier, D.W., Boisvert, R.F. and Clark, C.W., editors. (2010). NIST Handbook of Mathematical Functions. Cambridge University Press, New York, NY; http://dlmf.nist.gov/

Press, W.H., Teukolsky, S.A., Vetterling, W.T. and Flannery, B.P. (2007). Numerical Recipes: The Art of Scientific Computing ( $3 r d$ ed.).Cambridge University Press, New York, NY; http://numerical.recipes/

Tseng, W.-K.,A. Earle, M.A. and Guo, J.-L. (2012). Direct and Inverse Solutions with Geodetic Latitude in Terms of Longitude for Rhumb Line Sailing. The Journal of Navigation, 65, 549-559.

Williams, R. (1998). Geometry of Navigation, Horwood Publishing Ltd, Chichester, UK.

Zevering, K.H. (2006). Dependability of Position Solutions in Celestial Sight-Run-Sight Cases - Part 1. The Journal of Navigation (2006), 59, 155-166; The Running Fix Technique Response To G. Huxtable's Comments The Journal of Navigation (2006), 59, 521-529. 